\title{
Mismatch Negativity is a Sensitive and Predictive Biomarker of Perceptual Learning During Auditory Cognitive Training in Schizophrenia
}

\author{
Veronica B Perez ${ }^{1,2,3}$, Melissa Tarasenko ${ }^{1,2}$, Makoto Miyakoshi' ${ }^{2}$, Sean T Pianka ${ }^{2}$, Scott D Makeig ${ }^{2}$, \\ David L Braff ${ }^{1,2}$, Neal R Swerdlow ${ }^{2}$ and Gregory A Light ${ }^{*, 1,2}$ \\ 'VISN 22 Mental IIIness Research, Education \& Clinical Center (MIRECC), San Diego VA Healthcare System, San Diego, CA, USA; ${ }^{2}$ Department \\ of Psychiatry, University of California, San Diego, San Diego, CA, USA; ${ }^{3}$ California School of Professional Psychology, Alliant International University, \\ San Diego, CA, USA
}

\begin{abstract}
Computerized cognitive training is gaining empirical support for use in the treatment of schizophrenia (SZ). Although cognitive training is efficacious for SZ at a group level when delivered in sufficiently intensive doses (eg, 30-50 h), there is variability in individual patient response. The identification of biomarkers sensitive to the neural systems engaged by cognitive training interventions early in the course of treatment could facilitate personalized assignment to treatment. This proof-of-concept study was conducted to determine whether mismatch negativity (MMN), an event-related potential index of auditory sensory discrimination associated with cognitive and psychosocial functioning, would predict gains in auditory perceptual learning and exhibit malleability after initial exposure to the early stages of auditory cognitive training in SZ. MMN was assessed in N=28 SZ patients immediately before and after completing I h of a speeded time-order judgment task of two successive frequency-modulated sweeps (Posit Science 'Sound Sweeps' exercise). All SZ patients exhibited the expected improvements in auditory perceptual learning over the I h training period $(p<0.00 \mathrm{I})$, consistent with previous results. Larger MMN amplitudes recorded both before and after the training exercises were associated with greater gains in auditory perceptual learning $(r=-0.5$ and $r=-0.67$, respectively, p's $<0.0 \mathrm{I})$. Significant pretraining vs posttraining MMN amplitude reduction was also observed $(p<0.02)$. MMN is a sensitive index of the neural systems engaged in a single session of auditory cognitive training in SZ. These findings encourage future trials of $M M N$ as a biomarker for individual assignment, prediction, and/or monitoring of patient response to procognitive interventions, including auditory cognitive training in SZ.
\end{abstract}

Neuropsychopharmacology (2017) 42, 2206-22I3; doi:I0.1038/npp.2017.25; published online 22 March 20I7

\section{INTRODUCTION}

Neurocognitive impairment is one of the most immobilizing and treatment-resistant symptoms for patients with schizophrenia (SZ) and is a reliable predictor of poor community outcomes (Green, 1996; Mohamed et al, 2008). Computerized cognitive training and other procognitive interventions are gaining empirical support for use in the treatment of SZ (Keshavan et al, 2014; Wykes et al, 2011). Recent findings demonstrate that the neural substrates of cognitive dysfunction in SZ can be modified by auditory training (AT) interventions that leverage neuroplasticity-based learning mechanisms (Subramaniam et al, 2012). AT is an evidencebased computerized intervention that is designed to sharpen

*Correspondence: Dr GA Light, Psychiatry Service II6A, VISN-22 Mental Illness Research, Education \& Clinical Center (MIRECC), San Diego VA Healthcare System, University of California, 3350 La Jolla Village Drive, San Diego, CA 9216I, USA, Tel: +I 619543 2496, Fax: + 619543 1801, E-mail: glight@ucsd.edu

Received 13 December 2016; revised 12 January 2017; accepted 16 January 2017; accepted article preview online 31 January 2017 the acuity and fidelity of frequency and temporal information during auditory perceptual processing (Nahum et al, 2013). AT uses intensive, adaptive tasks that place increasing demands on early perceptual processes that drive 'bottomup' gains in higher-order cognitive functions. Studies of SZ patients who completed $40-50 \mathrm{~h}$ of AT demonstrated large effect size improvements in verbal learning and memory and global cognition (Fisher et al, 2009). Although AT is effective at the group level, individual responses vary considerably; as many as $45 \%$ of patients exhibit little or no benefit (Bell et al, 2001; Murthy et al, 2012) even after $100 \mathrm{~h}$ of training (Fisher et al, 2010). At this time, there are no known predictors of the effectiveness of this time- and resource-intensive training. It is therefore critical that new approaches to predicting and monitoring treatment response be developed to maximize patient response to treatment.

Most psychiatric interventions, including AT, are implemented in the absence of knowledge about individual variation in important domains of brain function that might influence therapeutic response and outcome. This 'one-sizefits-all' approach to treatment is problematic, with treatment 
failure occurring far too often and incurring substantial cost to the patient, family, therapist, and larger social system. To 'bend the curve' (Light and Swerdlow, 2015) on the individual outcomes and societal impact of chronic psychotic illness, we must advance our understanding of the neural substrates of the illness features that most strongly contribute to poor outcomes and use this information to guide mechanistically informed, personalized treatments (Perez et al, 2014; Tarasenko et al, 2016; Thomas et al, 2017).

Mismatch negativity (MMN) is a leading candidate biomarker for predicting and tracking response to novel procognitive treatment approaches, including AT (Draganova et al, 2009; Lovio et al, 2012; Paraskevopoulos et al, 2012). MMN is an event-related potential measure of auditory perceptual discrimination that is elicited in response to unattended, infrequent, deviant sound stimuli embedded in a sequence of frequently presented standard stimuli. Many previous studies have demonstrated MMN amplitude reduction at frontocentral electrodes in chronic $(d=1.00)$ (Light and Braff, 2005a, b; Salisbury et al, 2002; Shelley et al, 1991; Umbricht and Krljes, 2005), recent onset (Atkinson et al, 2012; Jahshan et al, 2012; Salisbury et al, 2002) and even unmedicated SZ patients (Light et al, 2015). MMN is suitable for use as a repeated measure in intervention studies as it shows stability over short and long retest intervals (12-month ICC $=0.90)$ (Light et al, 2012) and is unaffected by order or practice effects (Light and Braff, 2005b; Rissling et al, 2013). MMN accounts for substantial variance in verbal learning and memory (Light et al, 2007), psychosocial functioning (Light and Braff, 2005a, b), and level of independence in the community (Light and Braff, 2005a, b) - distal outcomes targeted by AT.

MMN is already considered a sensitive index of auditory perceptual discrimination (Naatanen et al, 1978) and central auditory system plasticity (Menning et al, 2000; Naatanen, 2008), the proximal mechanisms that are targeted by AT. A previous study of 10 healthy volunteers demonstrated that 3 weeks of intensive $(1.5 \mathrm{~h} /$ day $)$ training in auditory perceptual processing produced significant increases in MMN amplitude that persisted after the cessation of training (Menning et al, 2000), suggesting that MMN is a robust indicator of plastic reorganization of auditory cortical representations. This view of $\mathrm{MMN}$ as an index of brain plasticity is additionally supported by previous findings showing that MMN amplitude both predicts and corresponds to changes in language acquisition as well as musical training in nonpsychiatric individuals (for a review, see (Naatanen, 2008).

Translational animal models of learning have shown that AT is thought to follow a two-phase model of skill acquisition (Vinogradov et al, 2012) in which behavioral auditory discrimination improves very early in the course of training, with maximal gains evident after the first training session and smaller gains following subsequent sessions. More recent clinical studies of auditory perceptual learning in humans have also identified significant changes as demonstrated by sensory event-related potentials (ERPs) following a full course of training, and, importantly, these training-induced neural modulations predicted improvements in cognition (Anguera et al, 2013; Berry et al, 2010). Whereas shifts in electroencephalogram (EEG) amplitude correspond to behavioral improvements in perceptual tasks and project to global cognitive gains, little is known about which factors contribute to inter-individual variation underlying the treatment response, particularly in the initial phase of rapid improvement.

In the present study, MMN was recorded immediately before and after a single 1-h training session of auditory 'Sound Sweeps' exercises (Posit Science) to determine whether MMN is a sensitive and early index of the neural systems engaged by these exercises. Based on the adaptive nature of AT, we hypothesized that SZ patients would show significant behavioral improvements in auditory perceptual learning following training. Furthermore, as larger $\mathrm{MMN}$ amplitude in SZ patients is associated with better cognitive performance (Baldeweg et al, 2004; Jahshan et al, 2012; Javitt et al, 1995; Thomas et al, 2017; Wynn et al, 2010), we hypothesized that larger pretraining MMN amplitude would be associated with greater gains in behavioral measures of auditory perceptual learning.

It is conceivable that a cognitive training intervention such as AT would be expected to enhance/normalize MMN in SZ patients, and some evidence of neural remediation associated with cognitive improvement following training has already been demonstrated (Anguera et al, 2013; Menning et al, 2000). Alternatively, given that $\mathrm{MMN}$ is also sensitive to perceptual tuning (Naatanen et al, 1993), it is possible that a 1-h training session could impart a significant reduction in amplitude consistent with initial stages of sharpening/perceptual reorganization that accompanies the initial exposure of auditory perceptual training exercises. In fact, Gazzaley and colleagues (Berry et al, 2010) found significant reductions in sensory ERP amplitudes following perceptual training. If $\mathrm{MMN}$, alone or in combination with other biomarkers (eg, cABR (Tarasenko et al, 2014), P50 (Popov et al, 2011), gamma band (Popov et al, 2012)), is sensitive to the neural systems engaged by auditory perceptual training, future trials may be accelerated by biomarker-informed assignment to treatments. Indeed, MMN has already been demonstrated to be sensitive to the $N$-methylD-aspartate antagonist, memantine (Swerdlow et al, 2016a), suggesting malleability within the neural systems that regulate perceptual discrimination, even in chronic SZ patients. As such, we secondarily aimed to explore whether significant pretraining vs posttraining change in MMN amplitude would be detected.

\section{MATERIALS AND METHODS}

\section{Participants}

Twenty-eight SZ patients (Structured Clinical Interview for DSM-IV) were recruited from community facilities for participation in this study. Audiometry was used to ensure normal hearing with detection ability for $1000 \mathrm{~Hz}$ tones binaurally at a $40-\mathrm{dB}$ sound pressure level. Exclusion criteria included substance abuse/dependence within the past year, a history of significant medical or neurological illness, head injury-related loss of consciousness $>20 \mathrm{~min}$, and a positive urinalysis for recreational drug use. All patients and their data were included. Clinical symptoms were evaluated with the Scales for the Assessment of Negative Symptoms (SANS) and Positive Symptoms (SAPS). Table 1 displays demographic and clinical characteristics of the sample. The study was approved by the Institutional Review Board of UCSD (protocol \# 130453), and informed written consent was obtained from all participants. 
Table I Demographic and Clinical Characteristics of the Sample

\begin{tabular}{lcc}
\hline & $N$ & $\%$ \\
\hline Demographic data & & \\
Gender & 25 & 89.3 \\
$\quad$ Male & 3 & 10.7 \\
$\quad$ Female & & \\
Handedness & 24 & 85.7 \\
$\quad$ Right & 4 & 14.3 \\
$\quad$ Left & & \\
Nicotine & 14 & 50.0 \\
$\quad$ Yes & 14 & 50.0 \\
$\quad$ No & & 3.6 \\
Medication type & 1 & 85.7 \\
$\quad$ Typical & 24 & 10.7 \\
$\quad$ Atypical & 3 & \\
$\quad$ Both & & 10.1 \\
& 45.54 & 2.7 \\
Age, years, mean (SD) & 12.6 & \\
Education, years, mean (SD) & & \\
Clinical characteristics, mean (SD) & & \\
SAPS global symptom total & 5.11 & \\
SANS global symptom total & & \\
\hline
\end{tabular}

\section{Auditory Perceptual Training Exercises}

From the suite of training tasks used in our colleagues' previous studies of cognitive gains in SZ patients (Adcock et al, 2009; Fisher et al, 2009; Subramaniam et al, 2012), participants completed speeded auditory discrimination exercises ('Sound Sweeps'; http://www.positscience.com/ our-products/brain-fitness-program) designed to improve auditory perceptual learning. In this task, participants listened to a series of two successive tone sweeps (varying in frequency range and interstimulus interval (ISI)), and then indicated with two corresponding button presses whether the frequency increased or decreased within each tone, respectively (Fisher et al, 2009). The exercise contains 15 stimulus sets composed of combinations of base frequency $(500,1000$, and $2000 \mathrm{~Hz}$ ) and duration; subjects must first complete stimulus sets with longer-duration stimuli by demonstrating sustained successful performance at short ISIs (ie, $20 \mathrm{~ms}$ ) before stimulus sets with shorter-duration stimuli are made available. Auditory exercises were psychophysically adaptive: (1) parameters within each stimulus set required for an individual subject to maintain $80 \%$ correct performance were established; and (2) task difficulty increased and decreased systematically and parametrically with performance changes.

\section{Psychophysical Auditory Perceptual Processing Speed Measures}

After completing a practice block to ensure that participants understood the task, all subjects engaged in three 20-min blocks separated by 5 -min breaks. These exercises were comfortably tolerated by all subjects. Each subject's performance was monitored by recording the number of stimulus sets (measured as a percentage of all available stimulus sets) that were completed over the training. Higher values indicate that the subject reliably advanced through more of the stimuli content of the exercise (ie, became proficient on trials with progressively shorter frequencymodulated sweep durations); lower scores indicate that the subject remained at the easiest training levels (Adcock et al, 2009). Auditory perceptual learning gains for each subject were defined as the mean percentage of improvement in auditory perceptual learning speed on psychophysically smaller threshold differences in tone duration and frequency (Tarasenko et al, 2016).

\section{MMN Paradigm}

Auditory stimuli were presented to participants at $85 \mathrm{~dB}$ sound pressure level via Etymotic ER3-A insert earphones. The passive auditory oddball paradigm used to elicit MMN comprised a sequence of tones, of which $82 \%$ were standards $(50 \mathrm{~ms}, 1000 \mathrm{~Hz})$ and $18 \%$ were deviants $(6 \%$ per deviant type) that differed in stimulus duration $(100 \mathrm{~ms}, 1000 \mathrm{~Hz})$, frequency $(50 \mathrm{~ms}, 1100 \mathrm{~Hz}$ ), or both duration and frequency (ie, 'double-deviant' $100 \mathrm{~ms}, 1100 \mathrm{~Hz}$ ). The sequence of stimulus presentation was pseudorandomized such that no deviant was repeated in succession with a minimum of three standard tones between each deviant presentation. Each deviant was presented a total of 229 times; the standard stimuli included 3985 tones. All tones had 5-ms rise/fall times and were presented with a fixed 500-ms stimulus onset asynchrony. The total recording time was $\sim 40 \mathrm{~min} / \mathrm{session}$. Participants were instructed to ignore auditory stimuli while viewing a silent movie. MMN was recorded immediately prior to engagement in the auditory perceptual learning exercises and again within 1-h after AT was complete.

\section{EEG Data Acquisition and Preprocessing}

EEG data were recorded from 64 channels using a BioSemi ActiveTwo system (www.biosemi.com). Additional electrodes were placed on the mastoids and nose, and electrodes above and below the right eye and at the outer canthi of both eyes were used to record vertical and horizontal electrooculogram data and to correct EEG for eye movement and blink artifacts. Recorded voltages at analog-to-digital conversion for each electrode site were made relative to a common mode voltage based on the ActiveTwo's CMS/DRL feedback loop. EEG data were continuously digitized at $2048 \mathrm{~Hz}$ and down-sampled to $512 \mathrm{~Hz}$ and referenced offline to averaged mastoid electrodes. Continuous EEG data were parsed into 600-ms epochs ( -100 to $500 \mathrm{~ms}$ ) for each stimulus type. After Independent Component Analysis (ICA) artifact reduction and baseline correction (-100 to $0 \mathrm{~ms}$ prestimulus baseline), EEG epochs containing amplitudes exceeding $\pm 75 \mu \mathrm{V}$ in any frontocentral electrode (F3, Fz, F4, C3, Cz and $\mathrm{C} 4)$ were rejected. ERP averages for standards and each deviant type were generated separately, and the resultant difference waves were low-pass filtered at $20 \mathrm{~Hz}$ (zerophase shift, $24 \mathrm{~dB} /$ octave rolloff) to remove any residual high-frequency artifact, consistent with established methods (Light and Braff, 2005a). Confirmation of polarity inversion for MMN differences waves was performed at mastoid electrodes sites. Mean amplitudes of ERP peaks P50 
a

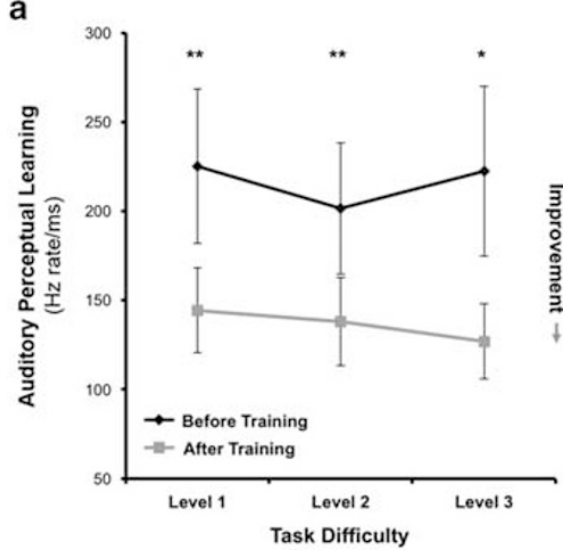

b

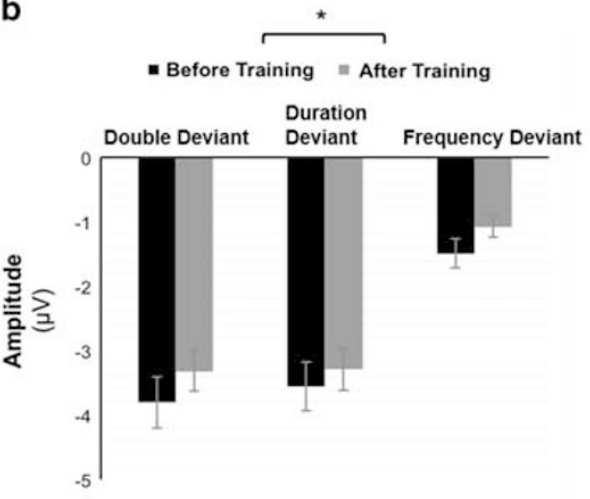

c

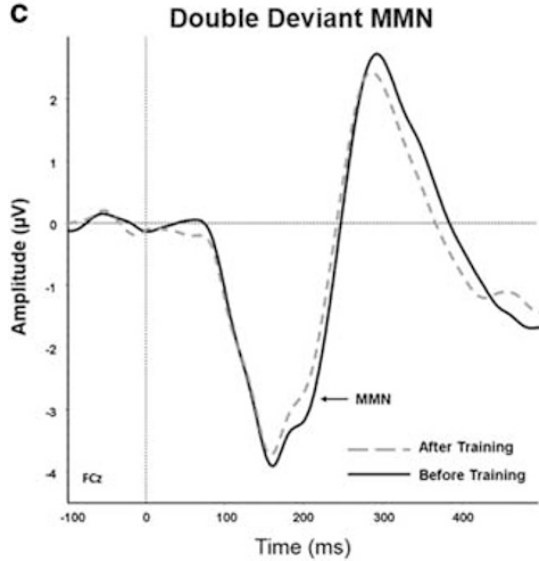

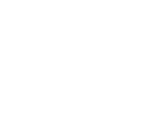

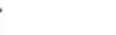

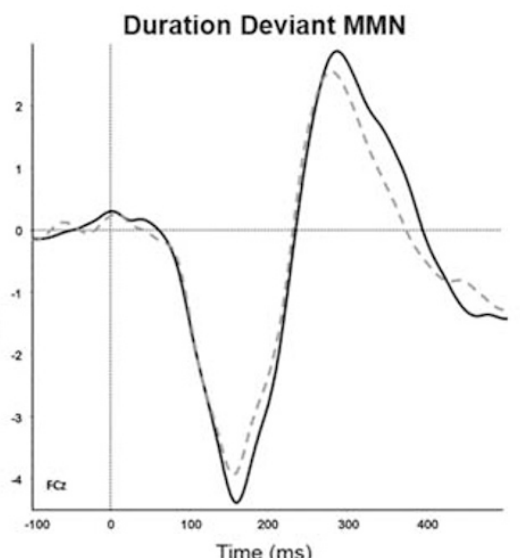

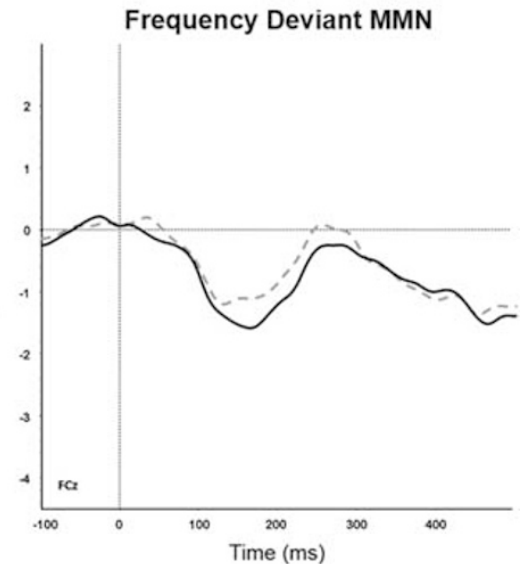

Figure I Behavioral and electrophysiological changes after auditory training. (a) Behavioral performance in auditory perceptual learning is shown across the first three levels of auditory training. Group means of baseline performance at each level are displayed in black; mean performance at the end of each training level is shown in gray. Auditory perceptual learning is plotted as tone threshold, where stimulus tone varied according to frequency modulation (FM) sweep rate $(\Delta \mathrm{Hz}$ rate)/tone onset asynchrony (ms). Auditory perceptual learning was significantly observed across each level of training. Lower thresholds imply greater auditory perceptual discrimination gains. (b) MMN at FCz is shown for double-, duration-, and pitch-deviant types. MMN amplitude after training (gray) was reduced relative to MMN before training (black). (c) MMN (standard-deviant tone difference waves) grand average waveforms before (solid) and after (dashed) auditory training are shown for double-, duration-, and pitch-deviant types. Waveforms for each deviant type are plotted from the FCz electrode. $* p<0.01$, *** $p<0.001$.

(40-80 ms) and N100 (80-120 ms) were calculated for each electrode in the standard stimuli. MMN amplitude was defined as the total mean area between 140 and $200 \mathrm{~ms}$ for each deviant type in the deviant-minus-standard difference waves.

\section{Statistical Analysis}

Behavioral performance. Changes in auditory perceptual learning after 1-h AT were assessed in the first three levels of the task, which all subjects completed. A repeated-measures analysis of variance (rmANOVA) assessed beginning and end point scores for each level of training difficulty (Level 1, Level 2 and Level 3) as within-subjects factors.

ERP analyses. ERP amplitude differences were assessed using a three-way rmANOVA with training (before, after), deviant type (duration, frequency, duration+frequency), and the 64 scalp electrodes as within-subjects factors. Greenhouse-Geisser nonsphericity correction was applied. Significant interactions were decomposed using planned $t$-tests. To examine the presence of neural-behavioral associations, Pearson's bivariate correlations assessed the relationships among pretraining and posttraining ERP components with behavioral gains in auditory perceptual learning following 1 -h training. ERP components (each deviant MMN at FCz, standard stimuli P50 and N100 at Cz) and behavioral gains in auditory perceptual learning were separately correlated with clinical (SAPS, SANS) factors.

\section{RESULTS}

\section{Behavioral Improvements in Auditory Perceptual Learning After Training}

Consistent with findings from perceptual learning studies in young adults (Karni and Sagi, 1993), older adults (Anguera et al, 2013; Berry et al, 2010), and patients with SZ (Fisher et al, 2009), significant training effects were evident in auditory perceptual learning (Figure 1a; $F(1,27)=623.02$, $p<0.001$ ), with no significant main effect of difficulty level $(p>0.9)$ or auditory perceptual learning-by-difficulty level interactions $(p>0.5)$. Follow-up paired $t$-tests showed significant training gains at each level (all $p<0.01$ ). Exploratory analyses of demographic and clinical characteristics, including 

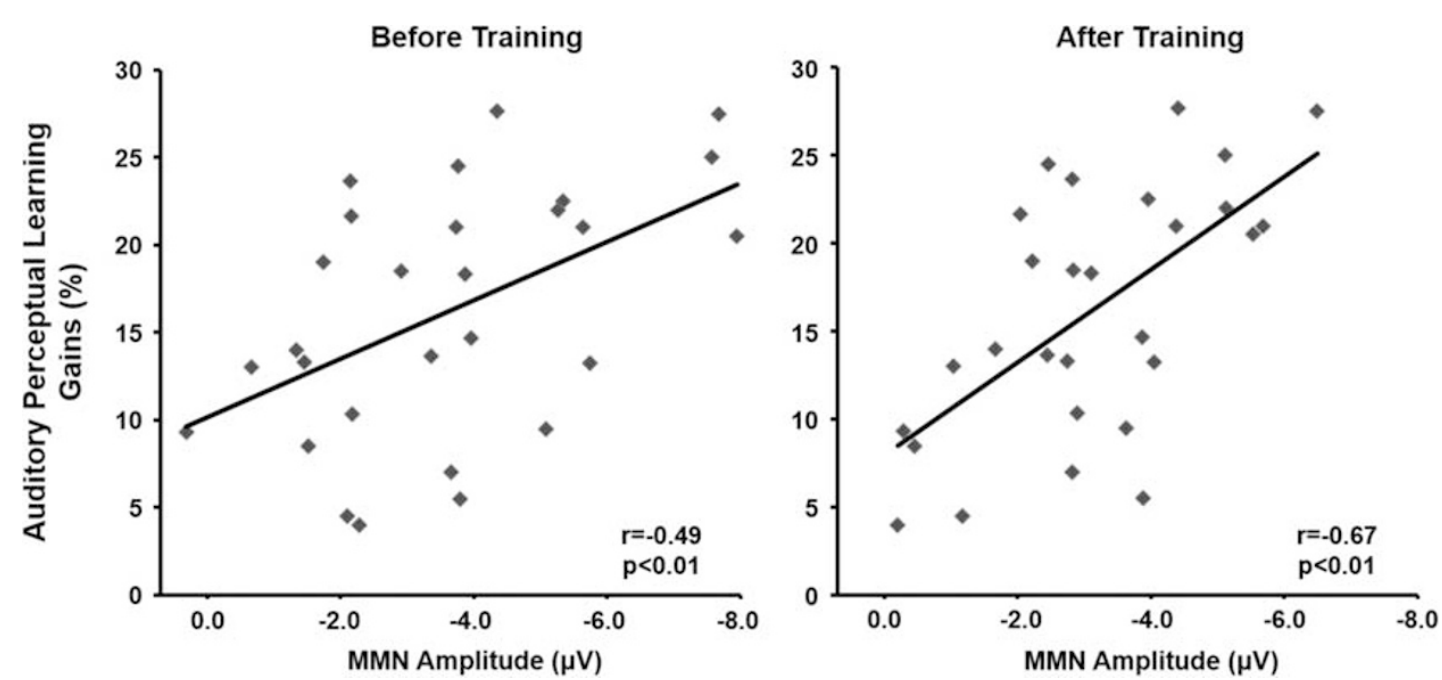

Figure 2 Mismatch negativity amplitude predicts gains in auditory perceptual learning. Mismatch negativity (MMN) amplitude at scalp channel FCz in response to duration+frequency double-deviants presented before and after auditory training (AT) is significantly associated with behavioral gains in auditory perceptual learning. Schizophrenia patients with the largest MMN amplitudes exhibited the largest behavioral improvements in response to I h of training. Behavioral improvement (\%) is the mean percentage change in baseline vs best score for each level.

age, gender, handedness, education, and positive or negative symptoms, failed to reveal significant associations with auditory perceptual learning gains.

\section{Pretraining vs Posttraining Differences in ERP Components}

MMN. MMN amplitude at baseline and post-AT (Figure 1b and c) revealed main effects of training $(\mathrm{F}(1,27)=6.5$, $p<0.02)$, deviant type $(\mathrm{F}(2,54)=67.8, p<.001)$, and electrode $(\mathrm{F}(63,1701)=78.3, p<0.001)$, as well as training $\times$ electrode $(\mathrm{F}(63,1701)=5.1, \quad p<.001)$ but not training $\times$ deviant $(\mathrm{F}(2,54)=0.07, p>0.9)$ interaction effects. Follow-up tests revealed that the training-related changes were only apparent at frontocentral electrodes. A slight but significant attenuation in MMN amplitude was observed (training: $(\mathrm{F}(1,27)=6.1, p=0.02$, Cohen's $d=0.25)$; MMN deviant type: $(\mathrm{F}(2,54)=61.1, p<.001)$ but no training $\times$ deviant type interaction $(p>0.6))$.

P50 and N100. Statistical assessment of both P50 and N100 amplitudes measured from the standard stimuli at baseline and post-AT revealed expected main effects of electrode $(p<0.001)$ but failed to detect significant training $(p>0.70)$ or training $\times$ electrode interaction effects (P50: $p>0.25$, N100: $p>0.10$ ). Follow-up tests confirmed that there were no training effects at any electrode site for P50 or N100 amplitudes (all p's $>0.30-0.99$ ).

\section{ERP Correlates of Auditory Perceptual Learning Gains After Training}

Confirming our hypothesis, larger auditory perceptual learning gains were significantly associated with larger (ie, more negative) pretraining double-deviant $(\mathrm{r}=-0.5$, $p<0.01$; Figure 2a) and duration-deviant $(\mathrm{r}=-0.45$, $p<0.02)$ but not the frequency-deviant $(r=-0.18$, n.s. $)$ MMN amplitudes at FCz. Additionally, posttraining double- deviant $\mathrm{MMN}$ at $\mathrm{FCz}$ accounted for $45 \%$ of the variance in auditory perceptual learning gains $(r=-0.67, p<0.01$; duration-deviant: $r=-0.59, \quad p<0.01$; pitch-deviant: $r=$ $-0.34, p=0.075)$. No significant associations were detected, however, between pretraining vs posttraining MMN difference scores and auditory perceptual learning gains. No significant associations with auditory perceptual learning gains were detected with P50 or N100 mean amplitudes at Cz in response to the standard stimuli.

\section{Relationship of Clinical Symptoms with Auditory Perceptual Learning Gains and EEG Activity}

Neither positive nor negative symptom subscale scores were significantly correlated with MMN at $\mathrm{FCz}$ for any deviant type before or after AT nor with P50 or N100 at Cz for standard stimuli before or after training. Similarly, neither positive nor negative symptom subscale scores significantly correlated with behavioral auditory perceptual learning gains after training. See Supplementary Material.

\section{DISCUSSION}

Computerized cognitive training and other cognitive interventions are gaining empirical support for use in the treatment of SZ. Although auditory cognitive training is efficacious for SZ at a group level when delivered in sufficiently intensive dosing, there is wide variability in individual responses, with some patients showing significant gains and others showing little or no benefit (Fisher et al, 2009; Murthy et al, 2012), underscoring the need for predictive biomarkers. To our knowledge, this is the first study to demonstrate that MMN, a well-validated and reliable translational biomarker, is sensitive to the neural systems engaged by a single 'dose' of auditory perceptual training exercises in SZ patients. Significant auditory perceptual learning was evident in all patients after 
completing 1-h of these exercises, consistent with previous results (Fisher et al, 2009; Subramaniam et al, 2012; Tarasenko et al, 2016). Both baseline and posttraining MMN amplitude, but not demographic or clinical variables or other auditory ERP components (ie, P50 or N100 amplitudes), significantly predicted the degree of behavioral improvement in auditory perceptual learning. These results support the continued development of biomarkers for the prediction of treatment response to cognitive training interventions in SZ patients.

The current study is not the first to demonstrate that MMN is sensitive to changes following a single session of AT. Naatanen et al (1993) previously demonstrated that plasticity of the auditory system in healthy subjects could be entrained by introducing more finely discrepant (ie, smaller and smaller) deviants over a single session, thereby 'sharpening' the precision for encoded auditory information. Gilbert (1994) also showed that perceptual learning is a robust phenomenon in which repeated exposure to a parametrically changing stimulus leads to a decrease in discrimination thresholds. In clinical populations, Lovio et al (2012) found larger baseline MMN amplitude predicted improvements in higher-order, cognitive functioning (eg, phonological processing, reading, and writing) after a single 3 -h session of training in dyslexic children. Together with our results, the convergent evidence from these studies demonstrates significant MMN malleability in response to just 1 -h of single-session perceptual training, even in chronic SZ patients.

There have been inconsistent findings in the direction of the neural change after low-level perceptual training. In an aging population, Berry et al (2010) reported a suppression of electrophysiological activity after training, albeit after a longer $(10 \mathrm{~h})$ training course. Likewise, fMRI studies have shown that, with extensive repetition of stimuli, neural adaptation was reflected via decreased brain activity over time (Miyakoshi et al, 2012). Consistent with these studies, but in contrast to Menning et al (2000) and Lovio et al (2012), the SZ patients in this study showed slight MMN amplitude reductions after training. Previous studies by de Villers-Sidani et al (2010) showed almost complete reversal of age-related auditory discrimation deficits in rats, demonstrating that auditory tuning is a plastic cortical filter shaped by operant training. In this context, AT appears to remediate a slowed and incomplete suppression of background distractors (de Villers-Sidani et al, 2010). Previously, we showed that both SZ patients and healthy subjects who suppressed $\mathrm{MMN}$ responses to task-irrelevant tones relative to a baseline recording while attending to a demanding visual CPT showed greater concurrent attentional functioning (Rissling et al, 2013). Taken together, mounting evidence supports the role of MMN as an indicator of malleability in auditory cortical processes with pretraining prediction of improvements in behavioral performance. Although baseline MMN predicting perceptual learning during auditory system training is encouraging, additional studies are needed to characterize the time course of MMN malleability over the course of treatment. It is conceivable that the initial reduction in MMN amplitude is a transient phenomenon indicative of the engagement of neuroplasticity-based mechanisms but that increases in amplitude emerge over the full course of treatment that accompany the expected improvements in cognition. Alternatively, it is possible that the reorganization of cortical networks associated with auditory system training may also change the cortical source contributions to scalp MMN and other ERP components. Thus future studies (eg, (Rissling et al, 2014)) using source localization and assessing changes in cortical contributions are also warranted.

Interventions that affect cortical plasticity may be useful for identification of cognitive training and rehabilitation strategies for patients with SZ. Our results support the view that EEG biomarkers can index auditory system training-induced changes, even in clinically symptomatic SZ patients. Although we would not expect a single hour of auditory perceptual training to produce enduring neuroplastic changes in the auditory cortical networks, we speculate that EEG measures obtained in the early stages of auditory system training (ie, pretraining, posttraining, or pretraining-posttraining MMN differences) might identify individuals who are sensitive to this type of training and conceivably predict procognitive gains following longer and established therapeutic protocols (Light and Swerdlow, 2014; Perez et al, 2014).

This study and the extant literature is still unable to answer the question of whether an individual patient is likely to experience a cognitive and functional benefit from this (or any other) resource-intensive intervention (Perez et al, 2014). One possibility is that patients with larger (ie, more similar to healthy control subjects) MMN responses will have the neural resources necessary to benefit from training (Keshavan et al, 2011; Light and Swerdlow, 2014), consistent with the biomarkers of health model (Light and Swerdlow, 2014). As with the present study, Kawakubo et al (2007) showed that larger pretreatment MMN predicted skills acquisition after a 3-month psychosocial training program. Alternatively, patients with more deficient MMN may have the most room for rapid improvement with a 'full dose' of training (Twamley et al, 2011).

The current results suggest an intriguing third possibility: Might 'auditory system training-sensitive' patients who demonstrate EEG malleability after an initial test dose of training-ie, a quantifiable degree of change in one or more cortical processes contributing to the scalp-recorded MMNalso be more likely to exhibit cognitive enhancement after a longer course of training? Studies are currently underway to answer these important questions. It is plausible that a biomarker profile of neural system functioning could ultimately be used to inform individual assignment to restorative and compensatory cognitive training interventions.

Experimental caveats here include the lack of experimental manipulation of medications and the absence of control groups and task conditions. Some medications may enhance or blunt training gains to the auditory exercises (Vinogradov et al, 2009). As AT has demonstrated effectiveness in a variety of populations, including neuropsychiatric groups and healthy subjects (HS), study enrollment was aimed to detect generalizable biomarker relationships in a heterogeneous sample of community-dwelling SZ patients. Considering the current study design, a traditional two-group (HS vs SZ) by two-condition (AT vs, eg, computer games) design would have confirmed whether SZ patients have deficits in MMN relative to HS; allowed for comparisons of the HS vs SZ slope of performance gains in auditory perceptual learning; demonstrated that the AT exercises improves auditory perceptual learning compared with 
control computer game conditions; and permitted HS vs SZ comparisons of the magnitude of the association between MMN and performance gains following training. Though these are scientifically important, at least some of the questions are currently addressed in the extant literature (Fisher et al, 2009; Shelley et al, 1991; Subramaniam et al, 2012) or will be examined in longer-term treatment studies.

It should be emphasized that a failure to benefit (however defined) from auditory system training could potentially arise from limitations in the particular cognitive program or aspects regarding its delivery (eg, dosing, schedule and platform) and not an inherent inability to benefit from this or any other cognitive remediation intervention. As suggested above, a lack of response to a full course of training does not indicate that a given patient would not benefit from other forms of cognitive remediation, alone or in combination with other interventions (Light and Swerdlow, 2014; Perez et al, 2014; Twamley et al, 2011). For example, AT plus vocational rehabilitation appears to be more effective than AT alone, as meta-analyses show, eg, (Wykes et al, 2011). Similarly, pharmacological augmentation of cognitive training (ie, 'PACT'; Swerdlow et al, 2016b) is a promising approach for accelerating the accrual of gains during AT. For example, we recently showed that when amphetamine, a pro-attention drug, was combined with a single 'dose' (1-h) of AT, synergistic gains in auditory perceptual learning relative to placebo $(p<0.02)$ was observed in antipsychotic-medicated SZ patients. Importantly, this learning was sustained for at least 1 week. This pattern of findings in SZ patients-immediate AT-induced changes in neurophysiological measures of MMN and PACT strategies toward the enhancement of auditory perceptual learning-provides a powerful opportunity to understand the mechanisms linking shifts in brain electrical activity and changes in higher-order cognitive processes. The finding of a neural-behavior relationship may be the first step upon which further studies that are designed to use biomarkers to deliver the 'right treatment' to the 'right patient' at the 'right time' may be predicated.

In conclusion, this proof-of-concept study demonstrates that MMN is an EEG biomarker with substantial theoretical and empirical links: (1) to the targeted mechanisms of AT (auditory perceptual learning and central auditory system plasticity); and (2) in a targeted population (SZ patients). These results suggest that MMN may also be a sensitive index of AT 'target engagement' that predicts initial response to training. Given the robust associations between MMN and neurocognitive and psychosocial functioning (Light and Braff, 2005a), this study provides support for further study of MMN as a predictive biomarker of (3) the targeted outcomes of AT, namely, improved cognitive and psychosocial functioning (Rissling et al, 2014; Thomas et al, 2017). The present results may accelerate future trials designed to use biomarkers to predict and monitor responses to a full course of training.

\section{FUNDING AND DISCLOSURE}

This study was supported by the Sidney R Baer, Jr. Foundation, the Brain and Behavior Research Foundation (NARSAD), Veterans Medical Research Foundation and the VISN-22 Mental Illness, Research, Education, and Clinical Center, the National Institutes of Mental Health (UL1TR000100, MH42228, MH065571, MH094151, MH093453, MH094320,
MH081944 and MH59803), and by a gift to SDM from The Swartz Foundation (Old Field, NY, USA). NRS is a consultant for Genco Sciences. GAL has served as a consultant for Boehringer-Ingelheim, Astellas, Heptares, Merck, Neuroverse, NeuroSig, and Takeda. The other authors declare no conflict of interest.

\section{REFERENCES}

Adcock RA, Dale C, Fisher M, Aldebot S, Genevsky A, Simpson GV et al (2009). When top-down meets bottom-up: auditory training enhances verbal memory in schizophrenia. Schizophr Bull 35: 1132-1141.

Anguera JA, Boccanfuso J, Rintoul JL, Al-Hashimi O, Faraji F, Janowich $J$ et al (2013). Video game training enhances cognitive control in older adults. Nature 501: 97-101.

Atkinson RJ, Michie PT, Schall U (2012). Duration mismatch negativity and P3a in first-episode psychosis and individuals at ultra-high risk of psychosis. Biol Psychiatry 71: 98-104.

Baldeweg T, Klugman A, Gruzelier J, Hirsch SR (2004). Mismatch negativity potentials and cognitive impairment in schizophrenia. Schizophr Res 69: 203-217.

Bell M, Bryson G, Greig T, Corcoran C, Wexler BE (2001). Neurocognitive enhancement therapy with work therapy: effects on neuropsychological test performance. Arch Gen Psychiatry 58: 763-768.

Berry AS, Zanto TP, Clapp WC, Hardy JL, Delahunt PB, Mahncke HW et al (2010). The influence of perceptual training on working memory in older adults. PLoS ONE 5: e11537.

de Villers-Sidani E, Alzghoul L, Zhou X, Simpson KL, Lin RC, Merzenich MM (2010). Recovery of functional and structural agerelated changes in the rat primary auditory cortex with operant training. Proc Natl Acad Sci USA 107: 13900-13905.

Draganova R, Wollbrink A, Schulz M, Okamoto H, Pantev C (2009). Modulation of auditory evoked responses to spectral and temporal changes by behavioral discrimination training. $B M C$ Neurosci 10: 143.

Fisher M, Holland C, Merzenich MM, Vinogradov S (2009). Using neuroplasticity-based auditory training to improve verbal memory in schizophrenia. Am J Psychiatry 166: 805-811.

Fisher M, Holland C, Subramaniam K, Vinogradov S (2010). Neuroplasticity-based cognitive training in schizophrenia: an interim report on the effects 6 months later. Schizophr Bull 36: 869-879.

Gilbert CD (1994). Learning. Neuronal dynamics and perceptual learning. Curr Biol 4: 627-629.

Green MF (1996). What are the functional consequences of neurocognitive deficits in schizophrenia? Am J Psychiatry 153: 321-330.

Jahshan C, Cadenhead KS, Rissling AJ, Kirihara K, Braff DL, Light GA (2012). Automatic sensory information processing abnormalities across the illness course of schizophrenia. Psychol Med 42: 85-97.

Javitt DC, Doneshka P, Grochowski S, Ritter W (1995). Impaired mismatch negativity generation reflects widespread dysfunction of working memory in schizophrenia. Arch Gen Psychiatry 52: 550-558.

Karni A, Sagi D (1993). The time course of learning a visual skill. Nature 365: 250-252.

Kawakubo Y, Kamio S, Nose T, Iwanami A, Nakagome K, Fukuda $\mathrm{M}$ et al (2007). Phonetic mismatch negativity predicts social skills acquisition in schizophrenia. Psychiatry Res 152: 261-265.

Keshavan MS, Eack SM, Wojtalik JA, Prasad KM, Francis AN, Bhojraj TS et al (2011). A broad cortical reserve accelerates response to cognitive enhancement therapy in early course schizophrenia. Schizophr Res 130: 123-129.

Keshavan MS, Vinogradov S, Rumsey J, Sherrill J, Wagner A (2014). Cognitive training in mental disorders: update and future directions. Am J Psychiatry 171: 510-522.

Light GA, Braff DL (2005a). Mismatch negativity deficits are associated with poor functioning in schizophrenia patients. Arch Gen Psychiatry 62: 127-136. 
Light GA, Braff DL (2005b). Stability of mismatch negativity deficits and their relationship to functional impairments in chronic schizophrenia. Am J Psychiatry 162: 1741-1743.

Light GA, Swerdlow NR (2014). Neurophysiological biomarkers informing the clinical neuroscience of schizophrenia: mismatch negativity and prepulse inhibition of startle. Curr Top Behav Neurosci 21: 293-314.

Light GA, Swerdlow NR (2015). Bending the curve on psychosis outcomes. Lancet Psychiatry 2: 365-367.

Light GA, Swerdlow NR, Braff DL (2007). Preattentive sensory processing as indexed by the MMN and P3a brain responses is associated with cognitive and psychosocial functioning in healthy adults. J Cogn Neurosci 19: 1624-1632.

Light GA, Swerdlow NR, Rissling AJ, Radant A, Sugar CA, Sprock J et al (2012). Characterization of neurophysiologic and neurocognitive biomarkers for use in genomic and clinical outcome studies of schizophrenia. PLoS ONE 7: e39434.

Light GA, Swerdlow NR, Thomas ML, Calkins ME, Green MF, Greenwood TA et al (2015). Validation of mismatch negativity and P3a for use in multi-site studies of schizophrenia: characterization of demographic, clinical, cognitive, and functional correlates in COGS-2. Schizophr Res 163: 63-72.

Lovio R, Halttunen A, Lyytinen H, Naatanen R, Kujala T (2012). Reading skill and neural processing accuracy improvement after a 3-hour intervention in preschoolers with difficulties in readingrelated skills. Brain Res 1448: 42-55.

Menning H, Roberts LE, Pantev C (2000). Plastic changes in the auditory cortex induced by intensive frequency discrimination training. Neuroreport 11: 817-822.

Miyakoshi M, Chen SH, Matsuo K, Wu CY, Suzuki A, Nakai T (2012). Extensive stimulus repetition leads older adults to show delayed functional magnetic resonance imaging adaptation. Brain Imaging Behav 6: 357-365.

Mohamed S, Rosenheck R, Swartz M, Stroup S, Lieberman JA, Keefe RS (2008). Relationship of cognition and psychopathology to functional impairment in schizophrenia. Am J Psychiatry 165: 978-987.

Murthy NV, Mahncke $\mathrm{H}$, Wexler BE, Maruff P, Inamdar A, Zucchetto $\mathrm{M}$ et al (2012). Computerized cognitive remediation training for schizophrenia: an open label, multi-site, multinational methodology study. Schizophr Res 139: 87-91.

Naatanen R (2008). Mismatch negativity (MMN) as an index of central auditory system plasticity. Int J Audiol 47 (Suppl 2): S16-S20.

Naatanen R, Gaillard AW, Mantysalo S (1978). Early selectiveattention effect on evoked potential reinterpreted. Acta Psychol (Amst) 42: 313-329.

Naatanen R, Jiang D, Lavikainen J, Reinikainen K, Paavilainen P (1993). Event-related potentials reveal a memory trace for temporal features. Neuroreport 5: 310-312.

Nahum M, Lee H, Merzenich MM (2013). Principles of neuroplasticity-based rehabilitation. Prog Brain Res 207: 141-171.

Paraskevopoulos E, Kuchenbuch A, Herholz SC, Pantev C (2012). Evidence for training-induced plasticity in multisensory brain structures: an MEG study. PLoS ONE 7: e36534.

Perez VB, Swerdlow NR, Braff DL, Naatanen R, Light GA (2014). Using biomarkers to inform diagnosis, guide treatments and track response to interventions in psychotic illnesses. Biomarkers Med 8: 9-14.

Popov T, Jordanov T, Rockstroh B, Elbert T, Merzenich MM, Miller GA (2011). Specific cognitive training normalizes auditory sensory gating in schizophrenia: a randomized trial. Biol Psychiatry 69: 465-471.
Popov T, Rockstroh B, Weisz N, Elbert T, Miller GA (2012). Adjusting brain dynamics in schizophrenia by means of perceptual and cognitive training. PLoS ONE 7: e39051.

Rissling AJ, Miyakoshi M, Sugar CA, Braff DL, Makeig S, Light GA (2014). Cortical substrates and functional correlates of auditory deviance processing deficits in schizophrenia. Neuroimage Clin 6: 424-437.

Rissling AJ, Park SH, Young JW, Rissling MB, Sugar CA, Sprock J et al (2013). Demand and modality of directed attention modulate 'pre-attentive' sensory processes in schizophrenia patients and nonpsychiatric controls. Schizophr Res 146: 326-335.

Salisbury DF, Shenton ME, Griggs CB, Bonner-Jackson A, McCarley RW (2002). Mismatch negativity in chronic schizophrenia and first-episode schizophrenia. Arch Gen Psychiatry 59: 686-694.

Shelley AM, Ward PB, Catts SV, Michie PT, Andrews S, McConaghy N (1991). Mismatch negativity: an index of a preattentive processing deficit in schizophrenia. Biol Psychiatry 30: $1059-1062$.

Subramaniam K, Luks TL, Fisher M, Simpson GV, Nagarajan S, Vinogradov S (2012). Computerized cognitive training restores neural activity within the reality monitoring network in schizophrenia. Neuron 73: 842-853.

Swerdlow NR, Bhakta S, Chou HH, Talledo JA, Balvaneda B, Light GA (2016a). Memantine effects on sensorimotor gating and mismatch negativity in patients with chronic psychosis. Neuropsychopharmacology 41: 419-430.

Swerdlow NR, Tarasenko M, Bhakta SG, Talledo J, Alvarez AI, Hughes EL et al (2016b). Amphetamine enhances gains in auditory discrimination training in adult schizophrenia patients. Schizophr Bull (e-pub ahead of print).

Tarasenko M, Perez VB, Pianka ST, Vinogradov S, Braff DL, Swerdlow NR et al (2016). Measuring the capacity for auditory system plasticity: an examination of performance gains during initial exposure to auditory-targeted cognitive training in schizophrenia. Schizophr Res 172: 123-130.

Tarasenko MA, Swerdlow NR, Makeig S, Braff DL, Light GA (2014). The auditory brain-stem response to complex sounds: a potential biomarker for guiding treatment of psychosis. Front Psychiatry 5: 142.

Thomas ML, Green MF, Hellemann G, Sugar CA, Tarasenko M, Calkins ME et al (2017). Modeling deficits from early auditory information processing to psychosocial functioning in schizophrenia. JAMA Psychiatry 74: 37-46.

Twamley EW, Burton CZ, Vella L (2011). Compensatory cognitive training for psychosis: who benefits? Who stays in treatment? Schizophr Bull 37 (Suppl 2): S55-S62.

Umbricht D, Krljes S (2005). Mismatch negativity in schizophrenia: a meta-analysis. Schizophr Res 76: 1-23.

Vinogradov S, Fisher M, de Villers-Sidani E (2012). Cognitive training for impaired neural systems in neuropsychiatric illness. Neuropsychopharmacology 37: 43-76.

Vinogradov S, Fisher M, Warm H, Holland C, Kirshner MA, Pollock BG (2009). The cognitive cost of anticholinergic burden: decreased response to cognitive training in schizophrenia. Am J Psychiatry 166: 1055-1062.

Wykes T, Huddy V, Cellard C, McGurk SR, Czobor P (2011). A meta-analysis of cognitive remediation for schizophrenia: methodology and effect sizes. Am J Psychiatry 168: 472-485.

Wynn JK, Sugar C, Horan WP, Kern R, Green MF (2010). Mismatch negativity, social cognition, and functioning in schizophrenia patients. Biol Psychiatry 67: 940-947.

Supplementary Information accompanies the paper on the Neuropsychopharmacology website (http://www.nature.com/npp) 